\title{
Effects of Wavelength Conversion on Self-healing Optical Networks
}

\author{
Hoyoung Hwang \\ Digital Media Engineering Dept., Anyang University \\ Anyang 430-714, South Korea \\ hyhwang@aycc. anyang.ac.kr
}

\begin{abstract}
This paper studies the effects of wavelength conversion on backup routing and spare capacity utilization in optical networks. The efficiency of spare wavelength utilization is proportionally increased as the wavelength conversion capability increases, different from the call blocking probability for which about $30 \%$ of wavelength conversion capability shows nearly the same performance as full wavelength conversion capability. The spare resource utilization efficiency can be improved by using alternate routing and wavelength assignment algorithms.
\end{abstract}

\section{Motivation}

Wavelength division multiplexing (WDM) optical networks can be implemented in all-optical manner without wavelength conversion, or in opaque manner with full wavelength conversion. A hybrid implementation is also possible with limited number of O-E-O conversion switches that provide partial wavelength conversion capability. In all-optical networks with only transparent switches, a single wavelength should be assigned to a lightpath throughout the optical route thus shows inflexibility in routing. The wavelength conversion capability can relieve this inflexibility by assigning different wavelengths to different links of an optical route. It is known that about $25-30 \%$ of partial or sparse wavelength conversion capability can provide nearly the same call blocking probability as that of full wavelength conversion networks [1]. However, the effects of wavelength conversion capability on the network protection/restoration aspects and on the spare resource utilization have not been studied enough.

This paper studies the effects of wavelength conversion on the spare resource utilization, and proposes an alternate backup connection provisioning method using semi-lightpath [2]. A set of one or more consecutive lightpaths to connect a source-destination pair is called semi-lightpath, and each lightpath segment to build a semi-lightpath is called sub-lightpath. An optical path can be divided into several sub-lightpath segments, and those sub-lightpath segments may have different wavelengths from others and can be shared among backup semi-lightpaths, thus wavelength conversion effect can be achieved. 


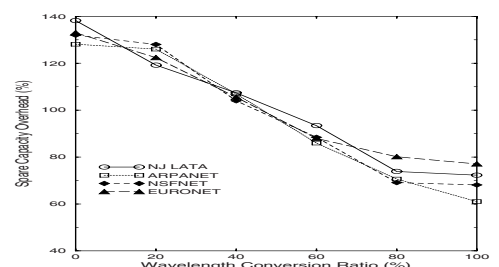

Fig. 1. Conversion ratio vs. Capacity overhead for single link failure

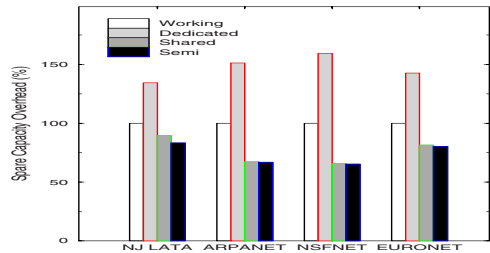

Fig. 2. Semi-lightpath vs. Other configurations

\section{Wavelength Conversion and Semi-lightpath}

Simulations were performed to see the effects of wavelength conversion on selfhealing optical networks. Four mesh topology networks are used assuming a network link has bidirectional fibers and each fiber had 40 wavelengths. Fig. 1 shows the spare capacity overhead versus wavelength conversion capability using disjoint shortest backup path provisioning. The wavelength overhead decreases almost linearly as the wavelength conversion ratio increases, which is different from the curve of call blocking probability mentioned earlier. This result comes from the probability of spare wavelength sharing according to the wavelength conversion capability.

In Fig. 2, the semi-lightpath based backup configuration was compared with dedicated capacity restoration in all optical networks and shared capacity restoration in opaque networks. The semi-lightpath configuration shows substantially better capacity efficiency compared with the dedicated path restoration, $51.1 \%$ to $94.1 \%$, and a little bit better capacity efficiency compared with the shared path restoration, $0.4 \%$ to $6.2 \%$. In this simulation, about $30 \%$ of network nodes were selected as wavelength conversion nodes based on 'higher degree node first' policy, and then updated manually considering even distribution in the network topology. Sub-lightpaths were configured between those selected nodes and the source/destination nodes of working lightpaths, and backup semilightpaths were configured to maximize the sharability of spare wavelengths on sub-lightpaths.

In summary, the semi-lightpath based backup path provisioning provides an improved way of spare wavelength utilization and routing, especially for hybrid optical networks with limited wavelength conversion capability. Analytical approach and detailed design method for semi-lightpath based backup path provisioning are to be studied.

\section{References}

[1] G. Shen et al. Operation of WDM Networks with Different Wavelength Conversion Capabilities. IEEE Communications Letters, 4(7):239-241, Jul. 2000. 207

[2] I. Chlamgtac et al. Lightpath Routing in Large WDM Networks. IEEE Journal on Selected Areas in Communications, 14(5):909-913, Jun. 1996. 207 